\title{
Fallo cardiaco agudo en el embarazo y puerperio
}

\author{
Acute heart failure in pregnancy and puerperium
}

Insuficiência cardíaca aguda na gravidez e no puerpério

\author{
José Miguel Jáuregui Solórzano (iD a,b, Miguel Alfredo Chung Sang Wong a,b,c, \\ Andrea Maily Velasco Miranda (D) a
}

\begin{abstract}
a Unidad de Cuidados Intensivos Maternos, Hospital GinecoObstétricoy Pediátrico Universitario de Guayaquil, Guayaquil-Ecuador: b Universidad de Especialidades Espíritu Santo, Guayaquil-Ecuador; c Universidad Católica de Santiago de Guayaquil, Guayaquil-Ecuador.
\end{abstract}

\section{Correspondencia a:}

osé Miguel Jáuregui Solórzano, jmjauregui25@hotmail.com

Recibido: 1 de mayo, 2021 Aceptado: 16 de mayo, 2021 Publicado: 1 de junio, 2021

\section{ARTÍCULO DE REVISIÓN}

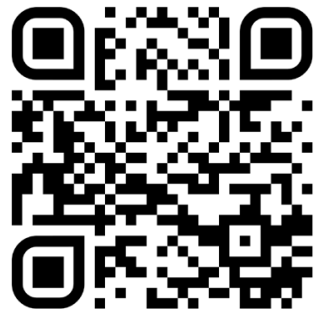

Escanea en tu dispositivo móvil o revisa este artículo en: https:// revistaclinicaguayaquil.org

\section{RESUMEN}

Las enfermedades cardiovasculares complican del 1 al 4\% de los embarazos y son la causa de morbimortalidad materna no obstétrica más común de los países desarrollados. Los cambios anatómicos y fisiológicos del sistema cardiovascular durante el embarazo pueden provocar insuficiencia cardiaca por exacerbación de condiciones prexistentes (cardiopatías congénitas) o llevar a desarrollar un nuevo estado patológico (cardiomiopatía periparto). La preeclampsia exacerba el riesgo de fallo cardiaco en pacientes cardiópatas. El síndrome de Takotsubo es una forma rara de miocardiopatía con fallo ventricular izquierdo que puede presentearse posterior a situaciones de estrés como el parto o cesárea. Por otro lado, el embolismo de líquido amniótico es una condición crítica con alta mortalidad materna. El manejo de fallo cardiaco dependerá de su causa, tiene aditamentos especiales como drogas deletéreas para el feto que vuelven a esta patología todo un reto para el intensivista obstétrico. Se describirán las patologías más importantes asociadas al fallo cardiaco agudo en el embarazo y puerperio.

Palabras clave: insuficiencia cardiaca; bienestar materno; periodo periparto; cardiomiopatía de Takotsubo; embolia de líquido amniótico

\begin{abstract}
Cardiovascular diseases complicate $1 \%$ to $4 \%$ of pregnancies and are the most common cause of non-obstetric maternal morbidity and mortality in developed countries. Anatomical and physiological changes in the cardiovascular system during pregnancy can cause heart failure due to exacerbation of pre-existing conditions (congenital heart diseases) or lead to the development of new conditions such as peripartum cardiomyopathy. Preeclampsia exacerbates the risk of heart failure in patients with preexisting heart disease. Takotsubo syndrome is a rare form of stress cardiomyopathy with left ventricular failure that can be observed after delivery or cesarean section. On the other hand, amniotic fluid embolism is a critical condition with high maternal mortality. The management of heart failure will depend on its cause, this management should consider special situations such as harmful drugs for the fetus that make these pathologies a challenge for the obstetric intensivist. The most important pathologies associated with acute heart failure in pregnancy and the puerperium will be described.
\end{abstract}

Key words: acute heart failure; maternal risk; peripartum cardiomyopathy; takotsubo; amniotic fluid embolism 


\section{RESUMO}

As doenças cardiovasculares complicam de $1 \%$ a $4 \%$ das gestações e são a causa mais comum de morbimortalidade materna não obstétrica em países desenvolvidos. Alterações anatômicas e fisiológicas no sistema cardiovascular durante a gravidez podem causar insuficiência cardíaca devido à exacerbação de condições pré-existentes (doença cardíaca congênita) ou levar ao desenvolvimento de um novo estado de doença (cardiomiopatia periparto). A préeclâmpsia exacerba o risco de insuficiência cardíaca em pacientes com doença cardíaca. A síndrome de Takotsubo é uma forma rara de cardiomiopatia de estresse com insuficiência ventricular esquerda que pode ser observada após o parto ou cesariana. Da mesma forma, a embolia de líquido amniótico é uma condição crítica com alta mortalidade materna. O manejo da insuficiência cardíaca dependerá da sua causa, pois possui acessórios especiais como medicamentos nocivos para o feto que tornam esta patologia um desafio para o intensivista obstétrico. As patologias mais importantes associadas à insuficiência cardíaca aguda na gravidez e no puerpério serão descritas.

Palavras-chave: insuficiência cardíaca aguda; risco materno; cardiomiopatia periparto; Takotsubo; embolia de líquido amniótico

\section{INTRODUCCIÓN}

A pesar que en los países latinoamericanos las principales causas de muerte materna son la hemorragia posparto y la sepsis, la enfermedad cardiovascular en la gestante presenta un grave problema debido al riesgo para la madre y el feto, es por ello, que este estudio cobra importancia. La mayor data de patología cardiaca se encuentra en trabajos realizados en el continente Europeo, en donde la Guía Europea destaca el manejo de enfermedades cardiovasculares durante el embarazo y reporta una tasa de admisión a Unidades de Cuidados Intensivos (UCI) por enfermedad cardiaca de 1 ingreso por cada 156 partos, y una mortalidad cercana al 5\%, convirtiéndose en la primera causa de muerte materna no obstétrica $(1,2)$. Estos valores han aumentado en el trascurso de los años, una de las causas son los embarazos en la edad reproductiva tardía, que pueden estar acompañados de factores de riesgos cardiovasculares como hipertensión arterial, obesidad y diabetes mellitus y, hoy en día, las mujeres con cardiopatías congénitas en su gran mayoría alcanzan la edad fértil generando aún mayor morbimortalidad materna y fetal (3).

\section{FISIOLOGÍA DEL EMBARAZO}

El embarazo produce cambios cardiovasculares expresados en mayor magnitud a partir de la mitad del segundo trimestre de gestación, caracterizados por expansión de volumen plasmático, incremento en la presión de llenado del ventrículo izquierdo, aumento del gasto cardiaco hasta en un $50 \%$ su valor basal y disminución de las resistencias vasculares periféricas debido al exceso de producción de óxido nítrico y la relaxina recuperando sus valores normales al cuarto día después del parto (4). Estos cambios hemodinámicos asociados a defectos cardiacos subyacentes ya intervenidos o de reciente diagnóstico "desenmascarados" durante el embarazo, patologías valvulares o a cardiomiopatías dilatadas idiopáticas pueden representar un alto riesgo de morbilidad y mortalidad para el feto y la madre (5).

\section{APROXIMACIÓN A LA EMBARAZADA CON SOSPECHA DE CARDIOPATÍA}

En la exploración física de una embarazada los cambios fisiológicos pueden simular una enfermedad cardiaca siendo a menudo difícil su diagnóstico clínico, ya que una gestante en su último trimestre puede presentar edemas periféricos, taquicardia, disnea, ortopnea e, inclusive, un soplo sistólico sin que tenga esto relación con una cardiopatía latente. Además, pueden presentar signos de fallo cardiaco izquierdo y/o derecho, siendo el edema agudo pulmonar la principal forma de presentación durante el embarazo y el principal motivo de consulta de emergencia. Se debe tener en cuenta un diferencial de causas de edema pulmonar tales como miocardiopatías, valvulopatías, fallo diastólico hipertensivo y sepsis (5). La evaluación por sospecha de cardiopatía durante el embarazo debe incluir titulación de péptidos natriuréticos, un electrocardiograma y un ecocardiograma doppler transtorácico (figura 1) (6). 


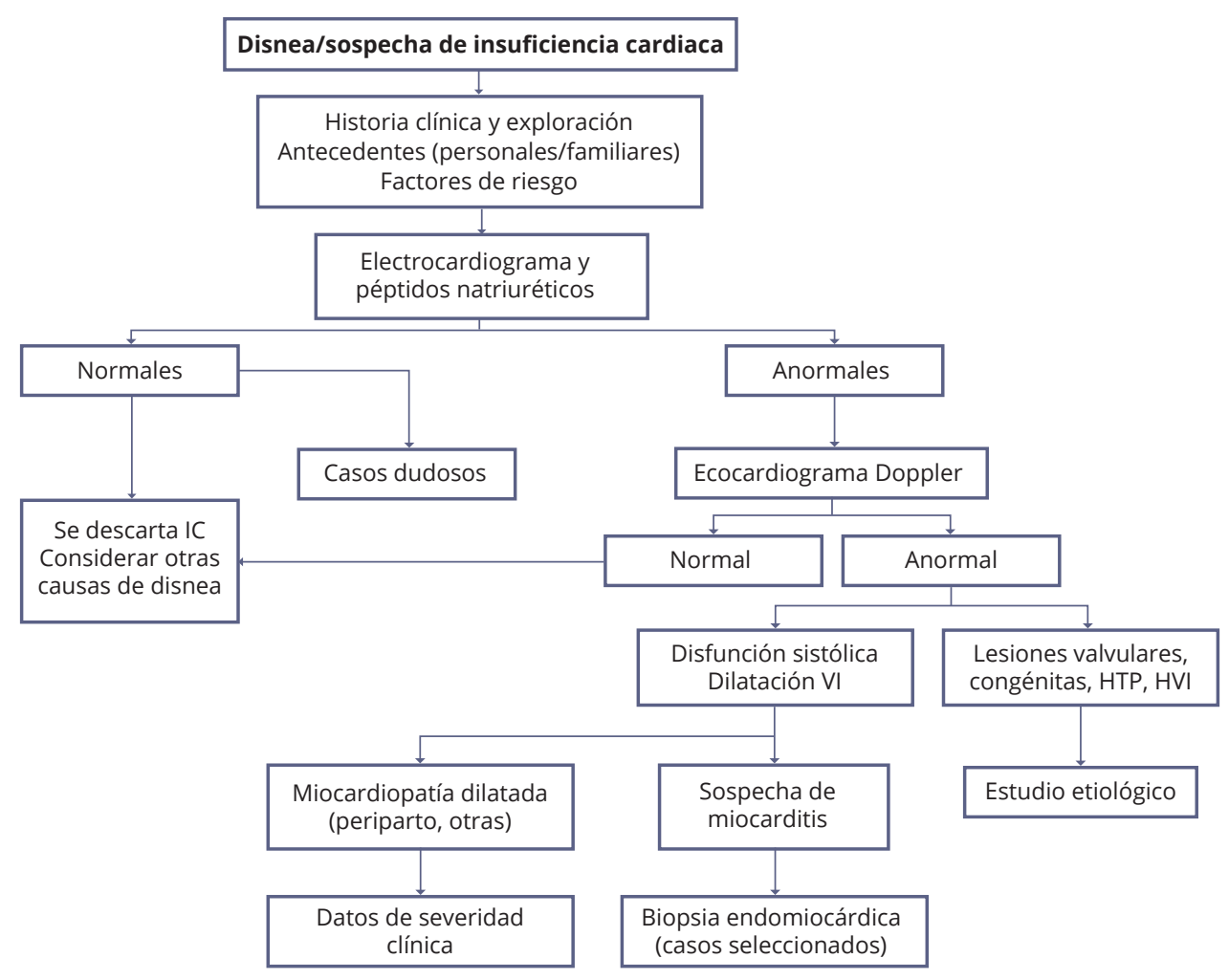

Figura 1. Algoritmo diagnóstico de insuficiencia cardiaca en el embarazo. HTP: hipertensión pulmonar, HVI: hipertrofia ventricular izquierda, IC: insuficiencia cardiaca, VI: ventrículo izquierdo. Tomado de Anguita et. al. Protocolo diagnóstico de la miocardiopatía en el embarazo. Medicine - Programa de Formación Médica Continuada Acreditado. 2017;12(43):2589-2592.

En toda embarazada con enfermedad cardiaca subyacente se debe evaluar el riesgo materno mediante predictores tales como la historia clínica, función ventricular y clase funcional NYHA, así como el puntaje CARPREG (CARdiac disease in PREGnancy). Actualmente, se acepta a nivel mundial que el riesgo materno sea evaluado según la clasificación modificada de la Organización Mundial de la Salud (OMS) descrita en la tabla 1.

Tabla 1. Clasificación modificada del riesgo cardiovascular materno según la OMS.

\begin{tabular}{|c|c|c|c|c|}
\hline & mOMS I & mOMS II & mOMS III & mOMS IV \\
\hline Patología & $\begin{array}{c}\text { Prolapso } \\
\text { valvular mitral, } \\
\text { estenosis } \\
\text { pulmonar leve, } \\
\text { defectos atriales } \\
\text { y ventriculares } \\
\text { corregidos }\end{array}$ & $\begin{array}{l}\text { Defectos atriales } \\
\text { o ventriculares } \\
\text { no corregidos, } \\
\text { tetralogía de Fallot } \\
\text { reparada, mayoría } \\
\text { de arritmias } \\
\text { supraventriculares }\end{array}$ & $\begin{array}{c}\text { Disfunción } \\
\text { ventricular } \\
\text { moderada (FE } \\
\text { 30-45\%), estenosis } \\
\text { aórtica severa } \\
\text { asintomática, } \\
\text { cardiopatía } \\
\text { cianosante no } \\
\text { reparada, dilatación } \\
\text { aórtica moderada, } \\
\text { cardiomiopatía } \\
\text { hipertrófica }\end{array}$ & $\begin{array}{c}\text { Hipertensión arterial } \\
\text { pulmonar, disfunción } \\
\text { ventricular severa } \\
\text { (FE <30\%), estenosis } \\
\text { mitral severa, } \\
\text { cardiomiopatía } \\
\text { periparto previa con } \\
\text { disfunción ventricular } \\
\text { residual, coartación } \\
\text { aórtica severa, } \\
\text { síndrome de Marfan } \\
\text { con dilatación aórtica } \\
>45 \text { mm }\end{array}$ \\
\hline Riesgo & Mínimo & Bajo & Alto & Extremadamente alto \\
\hline $\begin{array}{l}\text { Tasa de evento } \\
\text { cardiaco }\end{array}$ & $2.5-5 \%$ & $5.7-10.5 \%$ & $10-27 \%$ & $40-100 \%$ \\
\hline $\begin{array}{l}\text { Conducta/ } \\
\text { asesoramiento }\end{array}$ & $\begin{array}{l}\text { Continuar } \\
\text { embarazo }\end{array}$ & $\begin{array}{l}\text { Continuar } \\
\text { embarazo }\end{array}$ & $\begin{array}{l}\text { Embarazo } \\
\text { controlado por } \\
\text { experto }\end{array}$ & $\begin{array}{l}\text { Contraindicado } \\
\text { embarazo, considerar } \\
\text { interrupción }\end{array}$ \\
\hline $\begin{array}{l}\text { Seguimiento durante } \\
\text { el embarazo }\end{array}$ & Una o dos veces & 1 vez por trimestre & Bimensual & Mensual \\
\hline
\end{tabular}

FE: fracción de eyección. 


\section{ETIOLOGÍA Y FISIOPATOLOGÍA}

La falla cardiaca durante el embarazo puede ser debido a causas funcionales (arritmias, sobrecarga hídrica, efecto de drogas) y a causas estructurales. Los mecanismos principales son falla ventricular muscular directa, debido a cardiopatías congénitas, por valvulopatías o debido al incremento de las resistencias vasculares periféricas (7).

En el 2013, la Sociedad Europea de Cardiología publicó el European Registry on Pregnancy and Heart disease (Registry of Pregnancy And Cardiac disease, ROPAC), estudio observacional multicéntrico sobre la incidencia de enfermedades cardiovasculares que reunió
1321 embarazos con patologías cardiovasculares de 60 hospitales de 28 países, en un periodo de 3 años, cuyos resultados se observan en la figura $2(8,9)$. El $66 \%$ de los casos $(n=872)$ tenían una cardiopatía congénita subyacente y el $25 \%$, enfermedad valvular mitral o pulmonar. Desarrollaron fallo cardiaco agudo 173 mujeres (13.1\%), predominando como causa la presencia de cardiopatías congénitas en 71 pacientes, seguido de enfermedad valvular con 64 pacientes y miocardiopatías con 36 casos, de los cuales este último grupo fue el que tuvo mayor incidencia de fallo cardiaco agudo y mayor mortalidad $(8,9)$.

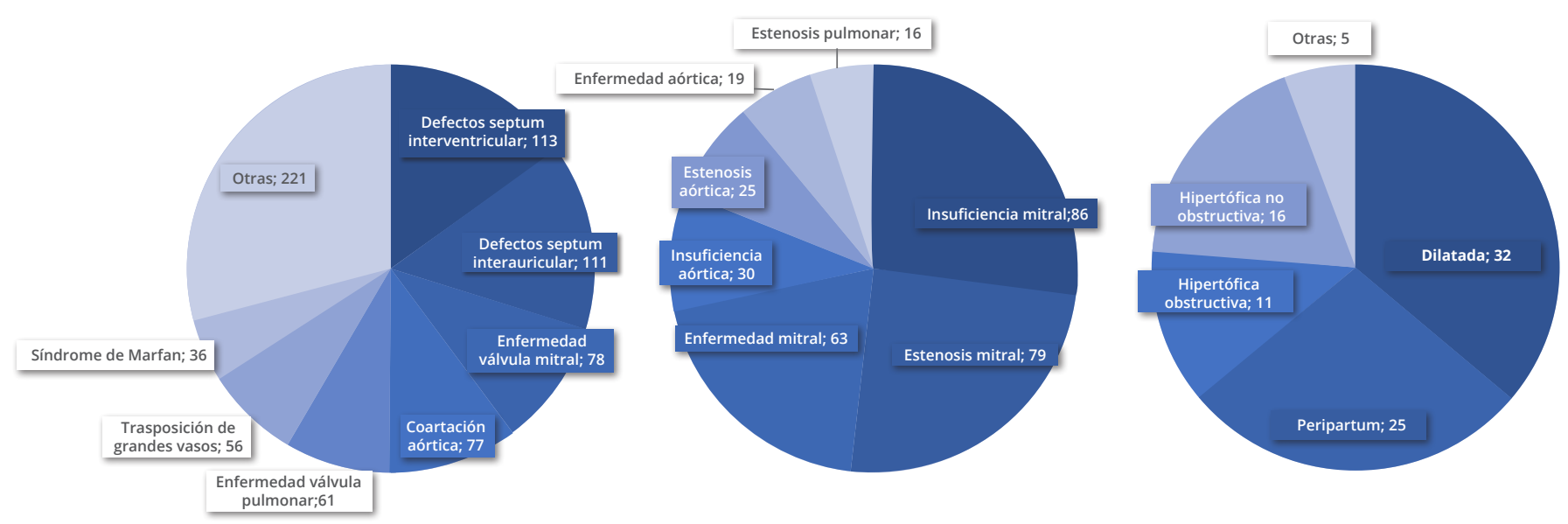

Figura 2. Enfermedades cardiovasculares por grupos según el estudio ROPAC, 2013. Adaptado de: Roos-Hesselink JW, Ruys TP, Stein JI, Thilén U, Webb GD, Niwa K, et al. Outcome of pregnancy in patients with structural or ischaemic heart disease: results of a registry of the European Society of Cardiology. Eur Heart J. 2013;34(9):657-65. doi: 10.1093/eurheartj/ehs270.

El episodio de fallo cardiaco se presentó en la semana 31 de gestación (IQ 23 - 40), y los picos de mayor incidencia se observaron entre la semana 23 y la 30, así como en los primeros días del puerperio. El fallo cardiaco desarrollado en el puerperio se asoció principalmente a miocardiopatía peripartum, insuficiencia mitral, otras miocardiopatías y tetralogía de Fallot (9). La mortalidad materna en el grupo con falla cardiaca fue del $4.5 \%$ versus el $0.5 \%$ del grupo sin falla cardiaca; en resultados fetales se observó alta incidencia de retardo de crecimiento intrauterino, mayor índice de cesáreas de emergencia y muerte fetal en $4.6 \%$ versus el $1.2 \%$ del grupo sin falla cardiaca (9). La adición de preeclampsia en una mujer con enfermedad cardiaca preexistente conlleva a falla cardiaca a más del 30\% de las pacientes (9).

En la estadística de pacientes ingresados en la UCI Materna del Hospital Universitario de Guayaquil, con un total de 724 ingresos entre los años 2017 y 2019, en los cuales se han diagnosticado 10 casos de cardiopatías (Figura 3), representando una incidencia del 1.4\%, comparable con a la prevalencia global. 


\section{Cardiopatías en el embarazo y \\ puerperio}

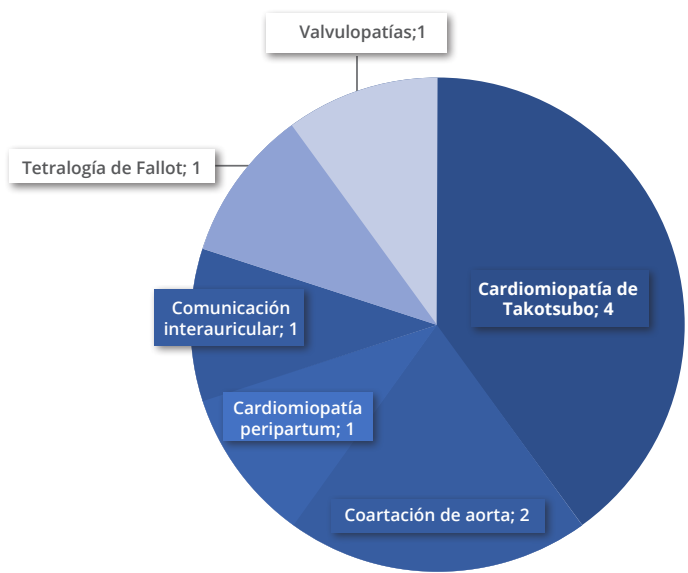

Figura 3. Cardiopatías diagnosticadas durante el embarazo y puerperio. Fuente: Base de datos de la UCI del Hospital Universitario de Guayaquil.

\section{Fallo cardiaco debido lesión miocárdica directa}

La cardiomiopatía es la causa más común de muerte durante y después del embarazo, siendo la patología más representativa la cardiomiopatía periparto (CMPP), seguida de las producidas por drogas, estrés, virus y embolismo de líquido amniótico.

\section{Cardiomiopatía periparto}

Considerada una enfermedad infrecuente, bien caracterizada, que se presenta en mujeres previamente sanas quienes desarrollan disfunción sistólica del ventrículo izquierdo con una fracción de eyección de menos del $45 \%$ durante el último mes de embarazo o dentro de 6 meses posteriores al parto, en ausencia de otras causas identificables de insuficiencia cardiaca $(1,10)$.

Actualmente se encuentra en curso un gran registro internacional prospectivo, que incluye 411 mujeres de 43 países; este ha demostrado que la CMPP ocurre a nivel mundial y afecta a todas las etnias y en distintas regiones del mundo, tanto en países desarrollados y en vías de desarrollo (10). La CMPP tiene una incidencia variable, que deriva de datos recogidos de estudios retrospectivos unicéntricos; puede ir desde 1:20000 en Japón, 1:4000 en Estados Unidos, o hasta 1:300 en Haití (11). Los factores predisponentes de la CMPP se exponen en la Tabla 2.
Tabla 2. Factores predisponentes de la cardiomiopatía peripartum

Ascendencia africana

Edad (> 30 y $<15$ años)

Preeclampsia

Multiparidad

Gestación múltiple

Obesidad, diabetes mellitus o hipertensión arterial preexistente

Tabaquismo, consumo de cocaína

Uso prolongado de tocolíticos (terbutalina) más de 4 semanas

\section{Etiología}

De etiología definitiva desconocida, se considera un origen de causa multifactorial, en el cual la activación anormal del sistema inmune desarrolla un papel fundamental. Existe además un trasfondo genético, agrupaciones familiares de CMPP presentan mutaciones que personas portadoras de cardiopatía dilatada idiopática tienen, especialmente en individuos con ascendencia genómica africana, en donde se ha detectado un genotipo GNB3 TT. Aquellas pacientes con este genotipo tienen mayor incidencia de falla ventricular izquierda y peores resultados en casos de CMPP (12). 
A nivel del sistema inmune durante el embarazo existe un aumento del estrés oxidativo, el cual genera elevación de los niveles de la proteasa catepsina $D$ que escinde a la prolactina en un fragmento angiostático 16KDA; esta proteína tiene efectos deletéreos sobre las células endoteliales vasculares causando apoptosis y disrupción de la función de los cardiomiocitos. Otras teorías de la lesión miocárdica en la CMPP incluyen el incremento de factores proinflamatorios como sFa/Apo-1, interferón gamma, IL-6 y TNF-alfa, infecciones virales como enterovirus (coxsackie y parvovirus 19), y la respuesta autoinmune con la producción de autoanticuerpos dirigidos a proteínas del cardiomiocito (13).

Los cambios hemodinámicos durante el embarazo pueden considerarse una causa, ya que existe el remodelamiento del ventrículo izquierdo debido al incremento del gasto cardiaco hasta en un $40 \%$, pudiendo haber una respuesta exagerada del ventrículo y provocar fallo sistólico.

\section{Diagnóstico}

El diagnóstico de CMPP se basa en un alto índice de sospecha, ya que puede presentarse dramáticamente con insuficiencia cardíaca aguda que requiere cuidados intensivos 0 puede desarrollarse sutilmente durante varias semanas. Rara vez se presenta antes de las 36 semanas de gestación, y aparece, en su mayoría, en el primer mes posterior al parto.

La presentación clínica es la típica que se observa en insuficiencia cardiaca con fallo sistólico izquierdo, y puede ser de instalación grave o paulatina, siendo ésta última confundida con alteraciones propias del embarazo tales como disnea y edema de miembros inferiores (14). Las pacientes presentan fatiga, palpitaciones, tos persistente, dolor precordial leve, y los casos más severos presentan ortopnea, hepatomegalia, derrames pleurales bilaterales y disnea de mínimos esfuerzos. Pueden existir signos clínicos de tromboembolismo pulmonar, debido a la formación de trombos en las cavidades cardiacas dilatadas (15).

El diagnóstico es de exclusión, el enfoque diagnóstico debe ir en busca de descarte de causas pulmonares y cardiovasculares que provoquen disnea e hipoxemia en el periparto y el puerperio (Tabla 3).

Tabla 3. Diagnóstico diferencial de cardiomiopatía peripartum.

\begin{tabular}{|c|c|c|}
\hline & Características & Pruebas diagnósticas \\
\hline $\begin{array}{l}\text { Cardiomiopatía dilatada } \\
\text { idiopática familiar (CMPDI) } \\
\text { desenmascarada en el } \\
\text { embarazo }\end{array}$ & $\begin{array}{l}\text { Mientras la CMPP se presenta en el } \\
\text { último mes, la CMPDI se presenta } \\
\text { a partir del segundo trimestre. La } \\
\text { CMPDI tiene mayores dimensiones } \\
\text { cardiacas que la CMPP. Historia } \\
\text { familiar positiva. }\end{array}$ & $\begin{array}{l}\text { Historia clínica, ECG, BNP, } \\
\text { ecocardiografía, test genético y } \\
\text { screening familiar. }\end{array}$ \\
\hline $\begin{array}{l}\text { Enfermedad valvular } \\
\text { preexistente desenmascarada } \\
\text { en el embarazo }\end{array}$ & Se presenta en el segundo trimestre. & $\begin{array}{l}\text { Historia clínica, examen físico, } \\
\text { ECG y ecocardiografía. }\end{array}$ \\
\hline $\begin{array}{l}\text { Enfermedad cardiaca congénita } \\
\text { preexistente no diagnosticada }\end{array}$ & $\begin{array}{l}\text { Debut en el segundo trimestre, } \\
\text { generalmente acompañada de } \\
\text { hipertensión pulmonar. }\end{array}$ & $\begin{array}{l}\text { Historia clínica, ECG, } \\
\text { ecocardiografía. }\end{array}$ \\
\hline $\begin{array}{l}\text { Enfermedad cardiaca } \\
\text { hipertensiva }\end{array}$ & Presenta disfunción diastólica. & Historia clínica, ecocardiografía. \\
\hline $\begin{array}{l}\text { Infarto de miocardio en } \\
\text { embarazo }\end{array}$ & Dolor precordial. & $\begin{array}{l}\text { ECG, enzimas cardiacas, historia } \\
\text { clínica, angiografía coronaria. }\end{array}$ \\
\hline Cardiomiopatía por VIH/SIDA & $\begin{array}{l}\text { Frecuentemente sin dilatación de } \\
\text { cámaras cardiacas. }\end{array}$ & Historia clínica, test VIH. \\
\hline
\end{tabular}

BNP: péptido natriurético cerebral, CMPDI: cardiomiopatía dilatada idiopática familiar, CMPP: cardiomiopatía peripartum, ECG: electrocardiograma, VIH: virus de la inmunodeficiencia humana.

Adaptado de: Sliwa, K., Hilfiker-Kleiner, D., Petrie, M., Mebazaa, A., Pieske, B., Buchmann, E., et al. Current state of knowledge on a etiology, diagnosis, management, and therapy of peripartum cardiomyopathy: a position statement from the Heart Failure Association of the European Society of Cardiology Working Group on peripartum cardiomyopathy. European Journal of Heart Failure. 2010;12:767-768. 
El electrocardiograma y ecocardiograma son esenciales en el diagnóstico de CMPP. En casos especiales pueden ayudar otras pruebas como el péptido natriurético auricular (BNP), resonancia magnética cardiaca, cateterismo cardiaco (cuando se sospeche de enfermedad coronaria aguda) y biopsia endomiocárdica.

El electrocardiograma es inespecífico, casi todos presentan anormalidades en la onda $T$ y segmento ST, además de ondas $\mathrm{Q}$ en precordiales septales y QRS ancho; también pueden existir signos de hipertrofia ventricular izquierda. Es una patología arritmogénica, sobretodo arritmias ventriculares. Su importancia radica en el diagnóstico diferencial de infarto de miocardio y embolismo pulmonar (16).

La radiografía de tórax muestra cardiomegalia, congestión pulmonar venosa y derrames pleurales, aunque para evitar la exposición a la radiación durante el embarazo este estudio puede ser reemplazado por la ultrasonografía pulmonar.

El ecocardiograma revela disfunción ventricular izquierda con una fracción de eyección (FEVI) de menos del $45 \%$ y, en ocasiones, disfunción cardiaca global con cámaras atriales y ventriculares dilatadas más trombo apical (Figura 4) (9). No todos los casos presentan un ventrículo izquierdo dilatado, pero un diámetro de más de $60 \mathrm{~mm}$ y una FEVI del $30 \%$ pronostican malos resultados (17). Como herramienta de seguimiento, el ecocardiograma debe ser realizado al alta, a las 6 semanas, a los 6 meses y al año, para determinar los cambios estructurales y funcionales del corazón.
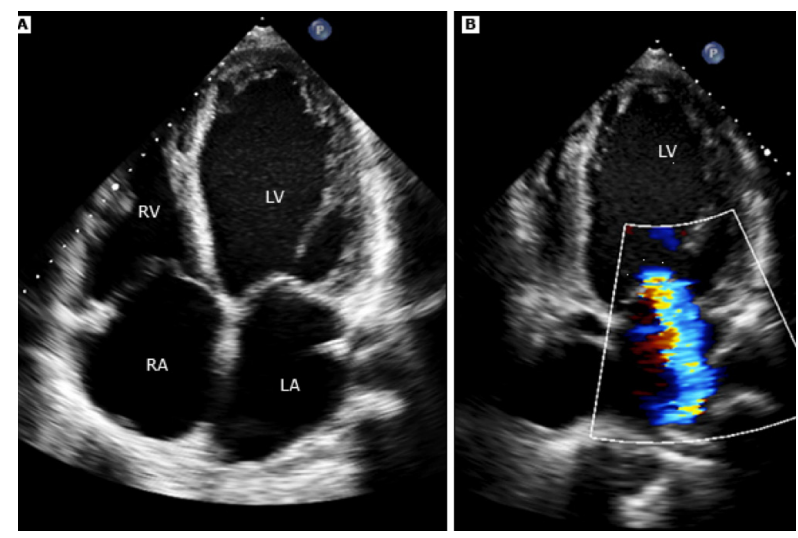

Figura 4. A. Vista apical de cuatro cámaras obtenida en un paciente con miocardiopatía periparto. Se observa dilatación de las cuatro cámaras cardíacas. B. Obsérvese, en el mismo paciente, un chorro Doppler a color de insuficiencia mitral moderada. Tomado de: Tsang W, Lang RM. Peripartum cardiomyopathy: Etiology, clinical manifestations, and diagnosis. UpToDate. 2020.
El papel de la resonancia magnética cardiaca necesita más estudios; permite una mejor evaluación de los volúmenes cardiacos, la función ventricular y la presencia de trombos comparado con la ecocardiografía. En cuanto a la resonancia magnética, debido a que no hay exposición a la radiación, las pruebas en serie se pueden realizar de manera segura en la mayoría de las pacientes con CMPP (18). Pero, cabe destacar, que no se recomienda la administración de gadolinio durante el embarazo, aunque es aceptable en mujeres lactantes (18). Pequeñas series han observado que la presencia del realce tardío del gadolinio puede estar relacionado con la recuperación de la función cardiaca.

\section{Tratamiento}

La historia natural de la enfermedad incluye desde casos de insuficiencia cardiaca leves, que resuelven con tratamiento médico y recuperando su función en un $50 \%$ de los casos, hasta choque cardiogénico con rápido deterioro sistémico y muerte (18).

El manejo de la cardiomiopatía periparto es similar al tratamiento de la insuficiencia cardiaca aguda, se debe tener en cuenta que las drogas habituales usadas en falla cardiaca pueden tener efectos tóxicos sobre el feto y complicar la lactancia. Así también se debe evitar la compresión aortocava, por lo que se indica mantener una posición de decúbito lateral.

En la fase aguda, caracterizada por edema pulmonar e hipoxemia, se puede utilizar ventilación no invasiva con presión positiva al final de la espiración de 5 a $7.5 \mathrm{cmH}_{2} \mathrm{O}$ y, si no hay mejoría, se deberá iniciar ventilación mecánica invasiva, además de furosemida en bolos de 20 a $40 \mathrm{mg}$. Los diuréticos deben manejarse con precaución y usar las dosis mínimas necesarias, ya que producen disminución del flujo uteroplacentario. Se recomienda nitroglicerina (dosis inicial 20 ug hasta 200 ug/min) si la presión arterial sistólica es mayor a $110 \mathrm{mmHg}$. Agentes inotrópicos como dobutamina o levosimendán deben usarse cuando haya signos de baja perfusión tisular (por ejemplo, piel fría, acidosis, falla renal, encefalopatía) y en congestión pulmonar persistente, a pesar de diuréticos (11).

En casos con choque cardiogénico refractario a inotrópicos y a balón de contrapulsación intraaórtico se debe considerar implantar un dispositivo de asistencia ventricular mecánica como puente a la recuperación, al trasplante 
cardiaco o a la decisión (11). La anticoagulación es necesaria en falla sistólica con $\mathrm{FEVI}<35 \%$, se debe usar heparina sódica o heparina de bajo peso molecular.

Con respecto al parto, la inestabilidad hemodinámica y la hipoxemia que no mejoren con tratamiento médico serian indicativos de interrupción del embarazo por cesárea. Además, el tratamiento con diuréticos y betabloqueadores podría causar daño al feto, acelerando la indicación de interrupción del embarazo $(11,18)$. Se debe consultar con el anestesiólogo sobre técnica de sedación con anestesia general versus anestesia neuroaxial.

Una vez que la estabilidad hemodinámica se haya logrado, el tratamiento debe estar basado en diuréticos (usarse con precaución y en dosis mínimas necesarias), bloqueantes de los receptores adrenérgicos como atenolol (en periodos cortos parecería ser seguro para el feto), vasodilatadores periféricos como hidralazina, y digoxina, siempre monitorizando sus niveles para evitar toxicidad. Se debe evitar bloqueantes del eje renina-angiotensina-aldosterona (IECA, ARA II) por sus efectos nocivos sobre la función renal fetal, siendo éstos muy útiles en la etapa posterior al parto.

Arritmias ventriculares han sido reportadas en el $20 \%$ de las pacientes con CMPP, de preferencia usar sotalol (clase B de FDA), y en lo posible evitar amiodarona (clase D de FDA). Existe riesgo de muerte súbita en casos con $\mathrm{FEVI}<30 \%$ con clase funcional NYHA III - IV en los cuales se debe evaluar la necesidad de colocación de cardiodesfibrilador implantable (CDI). La terapia de resincronización cardiaca se puede considerar en casos en los que, a pesar de tratamiento médico, persiste en clases funcionales III IV con FEVI <35\% y QRS >120 mseg; ambas terapias deben ser exhaustivamente estudiadas previamente, debido a la historia natural de la enfermedad.

Con respecto al tratamiento con bromocriptina, supresor de la prolactina, algunos estudios sugieren que la adición de este fármaco al tratamiento farmacológico de la insuficiencia cardiaca puede ser beneficioso en el curso agudo de la CMPP. En un estudio abierto aleatorizado realizado por Sliwa et al. en Sudáfrica se comparó 10 pacientes en manejo con la bromocriptina a dosis de $2.5 \mathrm{mg}$ cada 12 horas por dos semanas seguidos de $2.5 \mathrm{mg} /$ día por 6 semanas versus terapia convencional, concluyendo que hay una tendencia a la mejoría de la fracción de eyección del ventrículo izquierdo del 27 al 58\%, en el grupo de mujeres que recibió la bromocriptina respecto del grupo que recibió la terapia convencional (19). La bromocriptina fomenta aún más el estado protrombótico, por lo que se requiere anticoagulación con esta terapia. No se han observado malformaciones fetales en tratamientos con bromocriptina (11). Aún faltan estudios de mayor tamaño que avalen su uso.

\section{Pronóstico}

Las pacientes con CMPP tienen un alto porcentaje de recuperación miocárdica comparado con otras cardiomiopatías no isquémicas. La mortalidad varía entre el 1 al 30\%, los factores determinantes son la clase funcional, la FEVI, la presencia de trombos intracavitarios y valores de marcadores inflamatorios elevados. Se sugiere que aquellas mujeres con signos de gravedad deben evitar embarazarse, ya que empeorarían su función cardiaca (18).

\section{Cardiomiopatía por estrés o de Takotsubo}

La miocardiopatía por estrés o de Takotsubo se considera una de las variantes peculiares de la miocardiopatía periparto, también llamado síndrome de abalonamiento apical. Se caracteriza por una disfunción transitoria y reversible de la región apical del ventrículo izquierdo, imitando un síndrome coronario agudo, pero en ausencia de enfermedad aterotrombótica coronaria, en el estudio angiográfico coronario están normales (20). El término takotsubo proviene de la similitud que tiene el ventrículo izquierdo debido al abalonamiento del ápex con las trampas para pulpos japonesas. Esta forma característica se observa en ecocardiograma y en ventriculografía invasiva.

Se presenta mayormente en mujeres, sobretodo en la menopausia, y ocurre en el $2 \%$ de los síndromes coronarios agudos con supradesnivel del segmento ST con troponinas positivas. A nivel de cuidados críticos se puede presentar hasta en un $20 \%$ recuperando su función sistólica casi en su totalidad a los 7 días (21).

\section{Etiología}

Su etiología es incierta, se le atribuye el exceso de catecolaminas endógenas (especialmente norepinefrina) que causan vasoespasmo y disfunción miocárdica. Esta patología se puede presentar en situaciones de estrés físico o emocional, o en contexto de procedimientos anestésicos y quirúrgicos (22), usualmente en 
personas mayores, pero también se ha descrito en mujeres experimentando parto por cesárea y en el periodo postparto inmediato (23).

\section{Presentación}

La presentación clínica es típica de fallo cardiaco (disnea, congestión pulmonar, taquiarritmias) $y$ en algunos casos puede causar choque cardiogénico. Relacionado a esto, se menciona el caso de una embarazada de 22 años que desarrolló eclampsia a las 32 semanas de embarazo y se le realizó parto por cesárea. A las 48 horas presentó signos de fallo cardiaco más hipotensión arterial, diagnosticándose choque cardiogénico, el electrocardiograma mostró ondas $T$ negativas en las derivaciones precordiales desde V2 hasta V6 y en D1 y aVL (Figura 5); en cuanto a los biomarcadores cardiacos, la troponina $\mathrm{T}$ estaba elevada y el proBNP en 20724 pg/ml; el ecocardiograma reveló dilatación del ventrículo izquierdo e hipocinesia del segmento medio y ápex del ventrículo izquierdo, fracción de eyección del $45 \%$, cabe resaltar que ecocardiograma fue realizado con dosis elevadas de inotrópicos, lo que sobreestima la FEVI de la paciente. Posterior a la fase aguda, se realizó seguimiento ecocardiográfico, el cual reportó mejoría de la función ventricular izquierda, con una FEVI del 60\%. En esta paciente no se realizó cinecoronariografía debido a la ausencia de factores de riesgo cardiovasculares, al ser reversible la disfunción ventricular se consideró cardiomiopatía de estrés, característica principal que la diferencia de una cardiomiopatía peripartum.

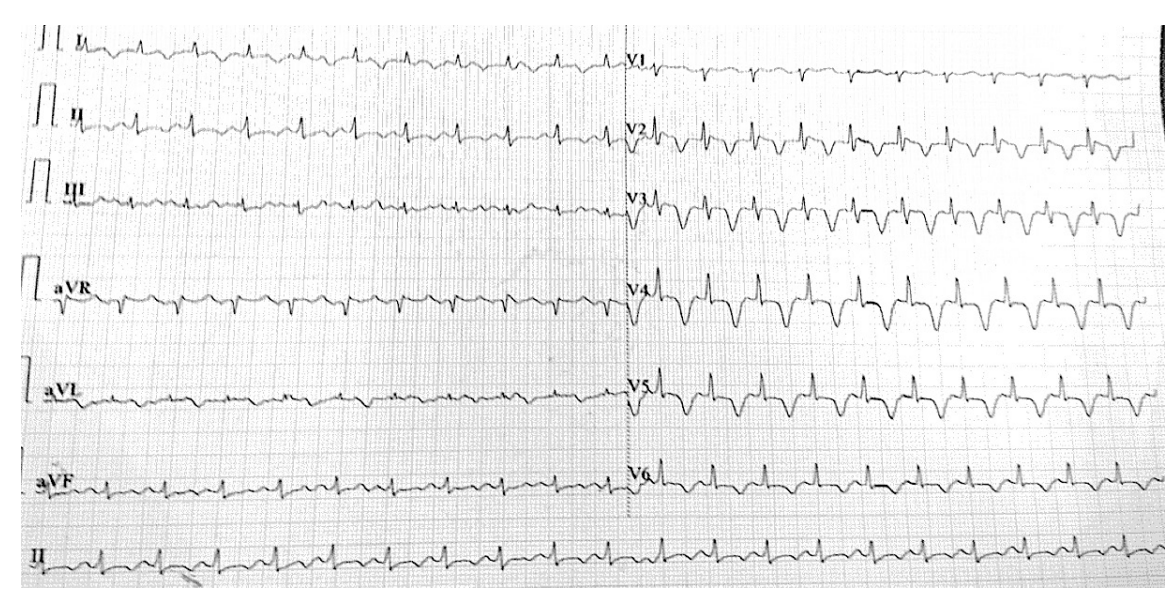

Figura 5. Electrocardiograma de 12 derivaciones en puérpera de 22 años, posterior a cesárea, que presentó cardiomiopatía de estrés. Fuente: Hospital Universitario de Guayaquil.

\section{Tratamiento y pronóstico}

El tratamiento del síndrome de Takotsubo es de soporte cardiovascular con drogas inotrópicas y vasopresores. Sin embargo, algunos casos ameritan ingreso a unidad de cuidados cardiocríticos y uso de balón de contrapulsación intraaórtico u otros dispositivos de asistencia circulatoria mecánica. Su pronóstico es favorable en casi todos los casos.

\section{Embolismo de líquido amniótico (ELA)}

El embolismo de líquido amniótico es una patología rara y catastrófica que ocurre en aquellos casos en que el líquido amniótico entra a la circulación materna durante el parto. Su incidencia reportada en Estados Unidos e Inglaterra es de 1 caso en 50000 partos.
La presentación clínica es fulminante, se presenta inmediatamente posterior al parto y se caracteriza por taquipnea e hipoxia, alteración del sensorio, hipotensión arterial y colapso cardiovascular, así como signos de coagulación intravascular diseminada (CID). Su fisiopatología no está clara, se describe una fase temprana con vasoespasmo pulmonar con disfunción ventricular derecha, seguido de una gran respuesta inflamatoria muy parecida a un choque anafiláctico que con lleva a una fase tardía con fracaso del ventrículo izquierdo y edema pulmonar. La mortalidad es alta, en la primera hora de instaurado el cuadro, el 50\% de los casos fallece (24).

Esta patología es un diagnóstico de exclusión, 
se debe diferenciar del tromboembolismo pulmonar, embolismo gaseoso, hemorragia, broncoaspiración, choque anafiláctico, sepsis, infarto de miocardio, miocardiopatía, eclampsia o reacciones transfusionales (24).

La Sociedad de Medicina Materno-Fetal (Society for Maternal-Fetal Medicine, SMFM) y la Fundación de Embolia de Líquido Amniótico (Amniotic Fluid Embolism Foundation) desarrollaron los criterios diagnósticos de ELA y se describen en la tabla 4.

Tabla 4. Criterios diagnósticos de embolia de líquido amniótico.

1. Arresto cardiaco súbito o hipotensión más hipoxia.

2. Confirmación de coagulación intravascular diseminada (CID).

3. Timing de presentación: durante el trabajo de parto o 30 minutos posteriores a salida de placenta.

4. Ausencia de fiebre $>38^{\circ} \mathrm{C}$ en el trabajo de parto.

El tratamiento es de mantenimiento, consiste en otorgar soporte hemodinámico y respiratorio, corregir la CID y prepararse para revertir hemorragia uterina hasta con histerectomía. No hay evidencia de los corticoides ayuden, salvo que haya una indicación estricta de su uso. Las sobrevivientes presentan secuelas neurológicas hasta en un $85 \%$ de los casos (24).

El diagnóstico con certeza es postmorten, en la autopsia se pueden observar células escamosas $y$ otros elementos fetales en los vasos pulmonares (25).

\section{CONCLUSIÓN}

El fallo cardiaco agudo en obstetricia es un escenario clínico que demanda un manejo multidisciplinario entre obstetras, cardiólogos e intensivistas, quienes deben trabajar en equipo para llevar a buen puerto a la madre y el feto. En los niveles primarios de atención es donde se debe realizar con suspicacia el examen físico de las gestantes, a fin de detectar a tiempo patologías cardiacas que compliquen la supervivencia materno fetal. Ante la sospecha clínica se deben realizar exámenes complementarios y filiar la patología cardiaca para poder asesorar el embarazo y planificar el momento y la forma del parto. Los eventos de descompensación de la función cardiaca al final del embarazo o al inicio del puerperio pueden tener distintos desenlaces, siendo el de mejor pronóstico el de la miocardiopatía por estrés. La miocardiopatía peripartum tiene una evolución impredecible, mientras que el embolismo de líquido amniótico es la complicación más temida en obstetricia, su reconocimiento es indispensable para un manejo oportuno.

\section{REFERENCIAS BIBLIOGRÁFICAS}

1. Regitz-Zagrosek V, Roos-Hesselink JW, Bauersachs J, Blomström-Lundqvist C, Cífková R, De Bonis M, et al. 2018 ESC Guidelines for the management of cardiovascular diseases during pregnancy. Eur Heart J. 2018;39(34):3165-3241. doi: doi. org/10.1093/eurheartj/ehy340.

2. Kuriya A, Piedimonte S, Spence A, Czuzoj-Shulman $\mathrm{N}$, Kezouh A, Abenhaim HA. Incidence and causes of maternal mortality in the USA.J Obstet Gynaecol Res. 2016;42(6):661-8. doi: 10.1111/jog.12954.

3. Elkayam U, Goland S, Pieper PG, Silverside CK. High-Risk Cardiac Disease in Pregnancy: Part I. J Am Coll Cardiol. 2016;68(4):396-410. doi: 10.1016/j.jacc.2016.05.048.

4. Hall M, George E, Granger J. El corazón durante el embarazo. Rev Esp Cardiol. 2011;64(11):10451050. doi: 10.1016/j.recesp.2011.07.009.

5. Sliwa K, Böhm M. Incidence and prevalence of pregnancy-related heart disease. Cardiovasc Res. 2014;101(4):554-60. doi: 10.1093/cvr/cvu012.

6. Anguita Sánchez M, Luque Moreno A, Paredes Hurtado N, Castillo Domínguez JC. Protocolo diagnóstico de la miocardiopatía en el embarazo. Medicine - Programa de Formación Médica Continuada Acreditado. 2017;12(43):2589-2592. doi: 10.1016/j.med.2017.10.015.

7. Anthony J, Sliwa K. Decompensated Heart Failure in Pregnancy. Card Fail Rev. 2016;2(1):20-26. doi:10.15420/cfr.2015:24:2.

8. Roos-Hesselink JW, Ruys TP, Stein Jl, Thilén U, Webb GD, Niwa K, et al. Outcome of pregnancy in patients with structural or ischaemic heart disease: results of a registry of the European Society of Cardiology. Eur Heart J. 2013;34(9):65765. doi: 10.1093/eurheartj/ehs270.

9. Ruys TP, Roos-Hesselink JW, Hall R, SubiranaDomènech $M T$, Grando-Ting J, Estensen $M$, et al. Heart failure in pregnant women with cardiac disease: data from the ROPAC. Heart. 2014;100(3):231-8. doi: 10.1136/ heartjnl-2013-304888.

10. Sliwa K, Mebazaa A, Hilfiker-Kleiner D, Petrie MC, Maggioni AP, Laroche C, et al. Clinical characteristics of patients from the worldwide registry on peripartum cardiomyopathy (PPCM): EURObservational Research Programme in conjunction with the Heart Failure Association of the European Society of Cardiology Study Group on PPCM. Eur J Heart Fail. 2017;19(9):1131-1141. doi: 10.1002/ejhf.780.

11. Sliwa K, Hilfiker-Kleiner D, Petrie MC, Mebazaa A, Pieske B, Buchmann E, et al. Current state of knowledge on aetiology, diagnosis, management, and therapy of peripartum cardiomyopathy: a position statement from the Heart Failure Association of the European Society of Cardiology Working Group on peripartum cardiomyopathy. 
Eur J Heart Fail. 2010;12(8):767-78. doi: 10.1093/ eurjhf/hfq120.

12. Sheppard R, Hsich E, Damp J, Elkayam U, Kealey A, Ramani G, et al. GNB3 C825T Polymorphism and Myocardial Recovery in Peripartum Cardiomyopathy: Results of the Multicenter Investigations of PregnancyAssociated Cardiomyopathy Study. Circ Heart Fail. 2016;9(3):e002683. doi: 10.1161/ CIRCHEARTFAILURE.115.002683.

13. Selle T, Renger I, Labidi S, Bultmann I, HilfikerKleiner D. Reviewing peripartum cardiomyopathy: current state of knowledge. Future Cardiol. 2009;5(2):175-89. doi: 10.2217/14796678.5.2.175.

14. Hilfiker-Kleiner D, Haghikia A, Nonhoff J, Bauersachs J. Peripartum cardiomyopathy: current management and future perspectives. Eur Heart J. 2015;36(18):1090-7. doi: 10.1093/ eurheartj/ehv009.

15. Kane A, Mbaye M, Ndiaye MB, Diao M, Moreira PM, Mboup C, et al. Évolution et complications thromboemboliques de la myocardiopathie idiopathique du péripartum au CHU de Dakar: étude prospective à propos de 33 cas [Evolution and thromboembolic complications of the idiopathic peripartal cardiomyopathy at Dakar University Hospital: forward-looking study about 33 cases]. J Gynecol Obstet Biol Reprod (Paris). 2010;39(6):484-9. French. doi: 10.1016/j. jgyn.2010.01.008.

16. Diao M, Diop IB, Kane A, Camara $S$, Kane A, Sarr M, Ba SA, Diouf SM. Enregistrement électrocardiographique de longue durée (holter) des 24 heures au cours de la cardiomyopathie idiopathique du péripartum [Electrocardiographic recording of long duration (Holter) of 24 hours during idiopathic cardiomyopathy of the peripartum]. Arch Mal Coeur Vaiss. 2004;97(1):2530.

17. Chapa JB, Heiberger HB, Weinert L, Decara J, Lang RM, Hibbard JU. Prognostic value of echocardiography in peripartum cardiomyopathy. Obstet Gynecol. 2005;105(6):1303-8. doi: 10.1097/01.AOG.0000161382.30233.ba.
18. Blauwet LA, Cooper LT. Diagnosis and management of peripartum cardiomyopathy. Heart. 2011;97(23):1970-81. doi: 10.1136/ heartjnl-2011-300349.

19. Sliwa K, Blauwet L, Tibazarwa K, Libhaber E, Smedema JP, Becker A, et al. Evaluation of bromocriptine in the treatment of acute severe peripartum cardiomyopathy: a proof-of-concept pilot study. Circulation. 2010;121(13):1465-73. doi: 10.1161/CIRCULATIONAHA.109.901496. Errata en: Circulation. 2010;121(21):e425. Struhman, Ingrid [corregido a Struman, Ingrid].

20. Citro R, Giudice R, Mirra M, Petta R, Baldi C, Bossone $\mathrm{E}$, et al. Is Tako-tsubo syndrome in the postpartum period a clinical entity different from peripartum cardiomyopathy? J Cardiovasc Med (Hagerstown). 2013;14(8):568-75. doi: 10.2459/ JCM.0b013e32835f1a49.

21. Gianni M, Dentali F, Grandi AM, Sumner G, Hiralal R, Lonn E. Apical ballooning syndrome or takotsubo cardiomyopathy: a systematic review. Eur Heart J. 2006;27(13):1523-9. doi: 10.1093/ eurheartj/ehl032.

22. Crimi E, Baggish A, Leffert L, Pian-Smith $M$, Januzzi J, Jiang Y. Acute reversible stress-induced cardiomyopathy with cesarean delivery un $\neg$ der spinal anesthesia. Circulation 2008;117:3052-3. doi: 10.1161/CIRCULATIONAHA.107.744102.

23. Kim SY, Yoon JH, Lee SH. Takotsubo-like severe left ventricular dysfunction after cesarean delivery in a 28-year old woman. Korean Circ J. 2011;41(2):1014. doi: 10.4070/kcj.2011.41.2.101.

24. Moore J, Baldisseri MR. Amniotic fluid embolism. Crit Care Med. 2005;33(10 Suppl):S279-85. doi: 10.1097/01.ccm.0000183158.71311.28.

25. Sinicina I, Pankratz H, Bise K, Matevossian E. Forensic aspects of post-mortem histological detection of amniotic fluid embolism. Int J Legal Med. 2010;124(1):55-62. doi: 10.1007/s00414009-0351-x. 


\section{ACERCA DE LOS AUTORES}

1. José Miguel Jáuregui Solórzano. Especialista en Medicina Crítica, Universidad de Especialidades Espíritu Santo de Guayaquil, Hospital Luis Vernaza. Médico staff del Servicio de Terapia Intensiva de Clínica Guayaquil. Tutor de Posgrado de Medicina Crítica de la Universidad Espíritu Santo, Guayaquil - Ecuador. Instructor del curso Fundamentos en Cuidados Críticos Obstétricos de la SCCM. Miembro de la Sociedad Ecuatoriana de Cuidados Intensivos. Clínica Guayaquil, Guayaquil-Ecuador. ORCID: 0000-0001-8405-5882

2. Miguel Alfredo Chung Sang Wong. Médico Clínico Intensivista, Universidad de Buenos Aires, Argentina. Profesor de posgrado de Medicina Crítica, Universidad de Especialidades Espíritu Santo; profesor de posgradodeGineco-Obstetricia, Universidad Católica Santiago de Guayaquil. Médico coordinador de la Unidad de Cuidados Intensivos Materna del Hospital Universitario de Guayaquil y Clínica Panamericana, Guayaquil Ecuador. Instructor de cursos SECl, SATI, SCCM, FEPIMTIC. Clínica Guayaquil, Guayaquil-Ecuador.

3. Andrea Maily Velasco Miranda. Médico residente senior de la Unidad de Cuidados Intensivos Materna del Hospital Universitario de Guayaquil, Ecuador.

ORCID: 0000-0002-0181-1951 